\title{
Vertical oscillation investigation of spatially elastically supported rigid plate - vehicle model
}

\author{
Blanka Skočilasová ${ }^{1, *}$, Jan Skočilas $^{2}$, František Klimenda ${ }^{1}$, Josef Soukup ${ }^{1}$, and Marian \\ Handrik $^{3}$ \\ ${ }^{1}$ UJEP in Usti nad Labem, FME, Institut of machines and energetic, Pasteurova 1, 40001 Ústí n. L, \\ Czech Republic \\ ${ }^{2}$ CTU in Prague, FME, Department of Process Engineering, Technicka 4, Prague 6, Czech Republic \\ ${ }^{3}$ UoZ in Zilina, Department of Applied Mechanics, Univerzitná 1, 01026 Žilina, Slovak Republic
}

\begin{abstract}
The article deals with introduction into the issue of the general asymmetry under vertical oscillation of systems of spatially elastically supported bodies with context of vehicle application. The various models are mentioned, quarter, half and full model (with different DOF) and advantages and disadvantages are discussed. The basic calculation procedures were presented for symmetry and asymmetry of geometry and excitation. The excitation is produced by jump change of plate support (analytically and numerically solved by Heaviside's function, in the experimental part by jump down from the wedges). The procedure to solve the basic 3D model is introduced for selected types of asymmetry of geometry and excitation. The practical approach is presented on the two-axle railroad vehicle. The vertical displacement, velocities, accelerations and angles of rotation of symmetry axes are considered. The time trend of the vertical displacement of the general point the aim of the calculation (3 DOF considered).
\end{abstract}

Keywords: vehicle model, symmetrical and asymmetrical model, vertical oscillation, displacement, kinematic excitation

\section{Introduction}

The long time broad attention has been pursued to problem solutions which deal with oscillation of the railway or road wheel vehicles assuming various hypothesizes, models, operation conditions. The extensive specialized literature [1] exists which is focused on this issues. What is missing is solution of the asymmetry effect at spatial vehicle models. Only particular solution of these issues are presented on the half geometry, planar, plane symmetry. For example when the plane symmetry of the model is assumed, only one longitudinal axis of vehicle is considered, where the displacement of the gravity center, various stiffness of supports, various intensity of viscous dumping or their combination is neglected. The quarter models are used for investigation of the vehicle oscillation the most often. They are usually

\footnotetext{
* Corresponding author: blanka.skocilasova@ujep.cz

Reviewers: Wiesława Piekarska, Krzysztof Talaśka
} 
vertically elastically bounded with dissipative elements with various number of bodies, so called models with more degree of freedom with bounded vertical displacements.

When the quarter model is applied two axes of vehicle symmetry are considered, i.e. total vehicle and kinematic excitation symmetry.

The investigation of the asymmetry effect on the vertical oscillation of the vehicle has to be performed by analysis of the various reasons and consequences on the spatial vehicle models.

Three basic cases of asymmetry has be distinguished with regard to axes of the geometrical symmetry. These axes are determined by mutually perpendicular symmetry axes of wheel-set and wheel-base of the vehicle and they are intersecting in the geometrical center of vehicle. These three cases are:

- asymmetry of the vehicle mass distribution with regard to axes of geometrical symmetry, - asymmetry of the elastic and dissipative elements distribution and their mechanical properties (assuming the linear bond of individual quantities and small displacements and rotation of system parts,

- asymmetry of the kinematic excitation, i.e. array of the surface roughness of the road (or railway), which defines kinematic excitation of the system in the contact place wheel-road (or wheel-rail).

These types of asymmetry can exists alone or together (more often case).

The first and till this time the most applied model has one of more degree of freedom, assumed as the one-dimensional chain of material points with elastic bonds, moving at one line or parallel lines, eventually with energy dissipation consideration (for example, [2, 3, 4] and others). The more accurate plane model is represented by system of two or more degree of freedom, where it has been assumed as plane chain of material points with elastic bonds or elastic supported "planar body" with inertia moment, again with energy dissipation consideration. The next step is three-dimensional model, represented by spatially supported body with two, three or generally six degree of freedom, or system of two of more bodies defined by mass and inertia moment, with six and more degree of freedom. E.g. the locomotive model has up to $270^{\circ}$ of freedom, see Rus [5], eventually again with energy dissipation consideration.

It is obvious that the basic condition for successful investigation of the vertical oscillation of the vehicle by experimental or theoretical (analytical, numerical, simulation) method is selection or definition of the suitable spatial model [1].

\section{Simple vehicle models}

For systematic analysis of various cases of asymmetry loading and oscillation it was necessary to defined simple but sufficiently general 3D model of the vehicle, satisfying required assumptions and tis variants.

The original designed mode contents the rigid plate which is supported at four springs supplied by two equal counterweights, see fig. 1. Counterweights allow to simulate various cases of the mass distribution and consequently position of the central inertia axes. The location of the weights on the plated affects the position of the gravity center $\mathrm{T}$ $\left(\bar{x}=e_{x}, \bar{y}=e_{y}, \bar{z}=0\right)$ of the three bodies system with regard to geometrical center $\mathrm{C}$ $(\bar{x}=0, \bar{y}=0, \bar{z}=0)$. The geometrical center lies on the axes intersection of wheel-set and wheel-base of the model, i.e. on axes of geometrical symmetry, by which the coordinate system is defined $(\bar{x}, \bar{y}, \bar{z})$. For solution the individual coordinate system is established $(x, y, z)$ with origin in the gravity center of system. Axes $\bar{x}, \bar{y}$ and $x, y$ lie in the same horizontal plane, vertical coordinates of the gravity center $\mathrm{T}$ and geometrical center $\mathrm{C}$ are in all cases equal $\bar{z}_{C}=\bar{z}_{T}=0$. In case of symmetrical system compounded by three bodies is 
position $\mathrm{C}=\mathrm{T}, e_{x}=0, e_{y}=0$ and main central axes of inertia are equal with axes of geometrical symmetry, and both coordinate systems are identical.

The model for analytic, numerical and simulation solution has been completed by dissipative element - viscous dumper, which is parallel to particular springs.

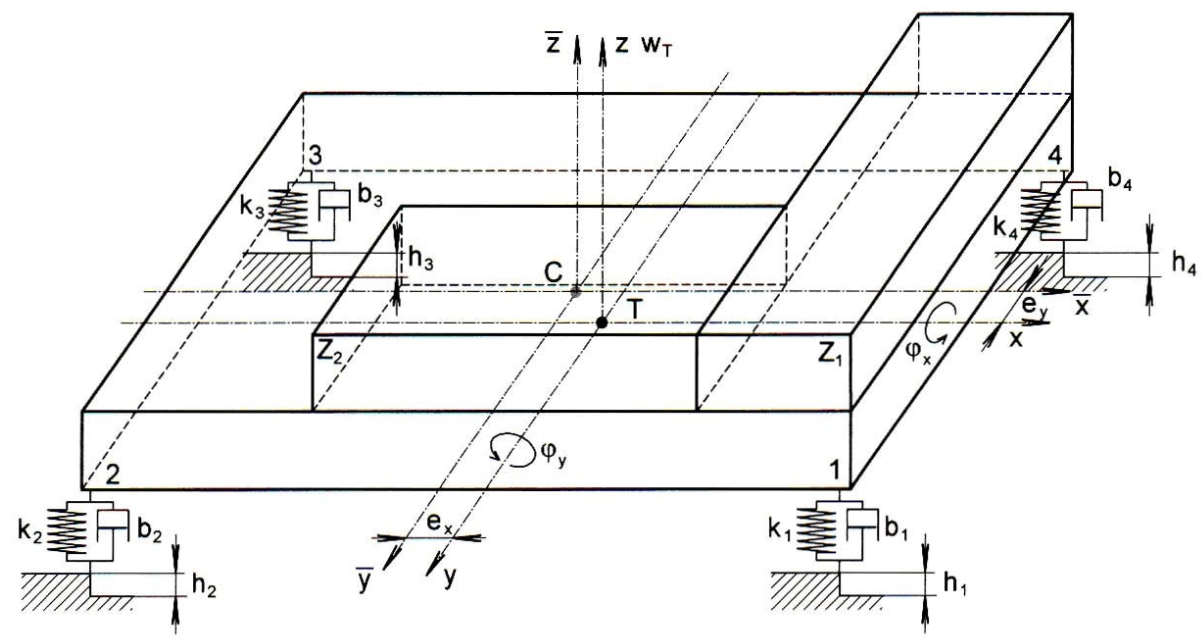

Fig. 1. Simple 3D vehicle model: 1, 2, 3, 4-position of the elastically dumping elements, $C$-geometrical center, $\mathrm{T}$ gravity center, $x, y, z$ - axes of symmetry, $k$ - spring stiffness, $b$ - dumping, $e_{x}, e_{y}$ - eccentricity of the gravity center under asymmetric loading, $\mathrm{h}$ - height of the roughness (kinetic excitation), w- vertical displacement, $\varphi_{x}, \varphi_{y}$ - rotations around axes of symmetry.

The criteria for comparison of the particular cases of asymmetry (loading and excitation) has been chosen time trend of the vertical displacement - the change of vertical coordinate of the gravity center $w_{T}(t)$, time trend of model rotation around central axes od inertia $\varphi_{x}(t)$ and $\varphi_{y}(t)$, and time trend of corresponding velocities $\dot{w}_{T}(t), \dot{\phi}_{x}(t), \dot{\phi}_{y}(t)$ and accelerations $\ddot{w}_{T}(t), \ddot{\phi}_{x}(t), \ddot{\phi}_{y}(t)$ components. These three components define time trend of displacements, velocities and accelerations in the arbitrary points $A\left(x_{A}, y_{A}\right)$ od system with three degrees of freedom, e.g. in the location of the support and consequently also wheel pressures on the bed (road or rail):

$$
\begin{aligned}
& w_{A}(t)=w_{T}(t)+x_{A} \phi_{y}(t)-y_{A} \phi_{x}(t), \\
& \dot{w}_{A}(t)=\dot{w}_{T}(t)+x_{A} \dot{\phi}_{y}(t)-y_{A} \dot{\phi}_{x}(t), \\
& \ddot{w}_{A}(t)=\ddot{w}_{T}(t)+x_{A} \ddot{\phi}_{y}(t)-y_{A} \ddot{\phi}_{x}(t),
\end{aligned}
$$

where $A=1,2,3,4$.

In the place of rigid plate support. i.e. in the points $A=1,2,3,4$ the coordinates $x, y$ are defined with regard to central axes of inertia, see Fig. 2.

$$
\begin{gathered}
x_{1}=l_{x 1}-e_{x}, \\
y_{1}=l_{y 1}-e_{y}, \\
x_{2}=-\left(l_{x 2}-e_{x}\right),
\end{gathered}
$$




$$
\begin{gathered}
y_{2}=l_{y 2}-e_{y}, \\
x_{3}=-\left(l_{x 3}+e_{x}\right), \\
y_{3}=-\left(l_{y 3}+e_{y}\right), \\
x_{4}=l_{x 4}-e_{x}, \\
y_{4}=-\left(l_{y 4}+e_{y}\right) .
\end{gathered}
$$

Vertical displacements in these points $A=1,2,3,4$ can be expressed due to roughness of the $\operatorname{road} h(x) \rightarrow h(t)$.

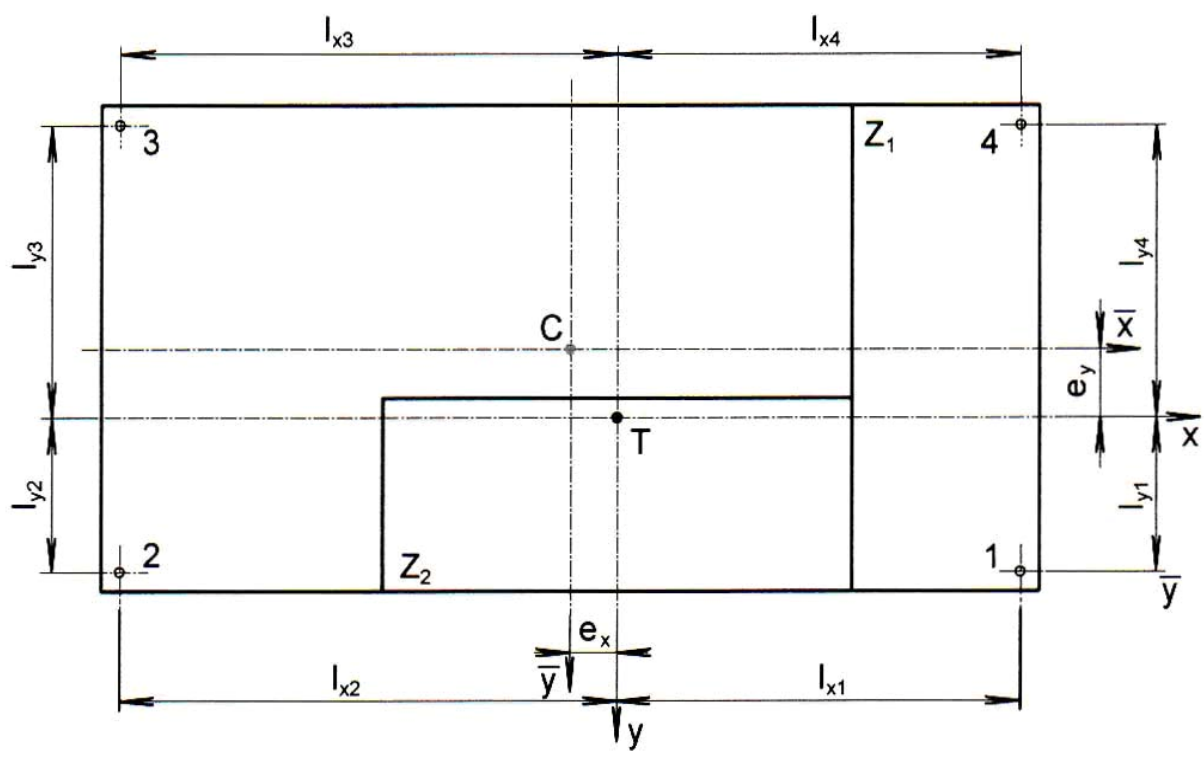

Fig. 2. Scheme of the model for vertical displacement assessment in the points $A=1,2,3,4$, $\mathrm{Z}_{\mathrm{i}}$ - weights (asymmetry simulation), $1_{\mathrm{x} i}, 1_{\mathrm{yi}}-$ distance between spring support place and axis of symmetry passing the gravity center $\mathrm{T}$

$$
\begin{aligned}
& w_{1}(t)=w_{T}(t)+\left(l_{x 1}-e_{x}\right) \varphi_{y}(t)-\left(l_{y 1}-e_{y}\right) \varphi_{x}(t)-h_{1}(t), \\
& w_{2}(t)=w_{T}(t)-\left(l_{x 2}+e_{x}\right) \varphi_{y}(t)-\left(l_{y 2}-e_{y}\right) \varphi_{x}(t)-h_{2}(t), \\
& w_{3}(t)=w_{T}(t)-\left(l_{x 3}+e_{x}\right) \varphi_{y}(t)+\left(l_{y 3}-e_{y}\right) \varphi_{x}(t)-h_{3}(t), \\
& w_{4}(t)=w_{T}(t)+\left(l_{x 4}-e_{x}\right) \varphi_{y}(t)+\left(l_{y 4}+e_{y}\right) \varphi_{x}(t)-h_{4}(t),
\end{aligned}
$$

where: $w_{x}(t)$ - displacement at point $1,2,3,4, w_{T}(t)$ - gravity center displacement, $l_{x i}, l_{y i}$ - distance between spring support place and axis of symmetry passing the gravity center $\mathrm{T}, e_{x}, e_{y}-$ eccentricity of the gravity center under asymmetric loading, $\varphi_{x}(t), \varphi_{y}(t)$ - rotations around axes of symmetry, $h_{x}(t)$ - height of the roughness (kinetic excitation).

The problem solution rests in the three function determination $w_{T}(t), \varphi_{x}(t)$ and $\varphi_{y}(t)$, those define displacement of the arbitrary point in time $t$, from motion equations of mechanical system with three degrees of freedom. The motion equation can be obtained from Lagrange's equations of second order: 


$$
\frac{d}{d t}\left(\frac{\partial E_{K}}{\partial \dot{q}_{j}}\right)-\frac{\partial E_{K}}{\partial q_{j}}+\frac{\partial E_{p}}{\partial q_{j}}+\frac{\partial R_{d}}{\partial \dot{q}_{j}}=Q_{j}, \quad \text { for } j=1,2, \ldots p
$$

where $p$ - number of degrees of freedom of system. In this case the definition of:

- kinetic energy is:

$$
E_{k}=\frac{1}{2}\left[m \dot{w}_{T}^{2}+J_{x} \dot{\phi}_{x}^{2}+J_{y} \dot{\phi}_{y}^{2}-2 D_{x y} \dot{\phi}_{x} \dot{\phi}_{y}\right]
$$

- potential energy is:

$$
E_{p}=\frac{1}{2} \sum_{j=1}^{4} w_{j}^{2} k_{j}=\frac{1}{2} \sum_{j=1}^{4}\left(w_{T}+x_{j} \phi_{y}-y_{j} \phi_{x}-h\right)^{2} k_{j},
$$

- Rayleigh's function of dissipation energy is :

$$
R_{d}=\frac{1}{2} \sum_{j=1}^{4} b_{j} \dot{w}_{J}=\frac{1}{2} \sum_{j=1}^{4} b_{j}\left(\dot{w}_{T}+x_{j} \dot{\phi_{y}}-y_{j} \dot{\phi_{x}}-\dot{h}_{j}\right),
$$

And vector of generalized of the excitation forces function $\mathbf{Q}_{j}$ and generalized coordinate $q_{j}$

$$
\boldsymbol{Q}_{j}\left(q_{j}, \dot{q}_{j}, t\right), \quad q_{j}=\left(w_{T}, \phi_{x}, \phi_{y}\right) .
$$

After derivation, the system of linear non-homogenous equations is obtained, expressed in the matrix form:

$$
\boldsymbol{M}_{h} \ddot{q}_{j}+\boldsymbol{M}_{b} \dot{q}_{j}+\boldsymbol{M}_{k} q_{j}=\boldsymbol{Q}_{j}\left(q_{j}, \dot{q}_{j}, t\right) \quad \text { for } j=1,2,3,
$$

where $\mathbf{M}_{h}$ - mass matrix, $\mathbf{M}_{b}$ - dumping matrix, $\mathbf{M}_{k}$ - stiffness matrix, $J_{x}, J_{y}$-inertia moments to axes $x, y, D_{i j}$ - deviation moments to appropriate plane.

The equation (6) can be arranged to form (after multiplication by diagonal matrix $\mathbf{D}=\left(d_{i j}\right)$ of third order from left) with Laplace's integral transformation:

$$
\boldsymbol{M} \ddot{q}_{j}(t)+\boldsymbol{B} \dot{q}_{j}(t)+\boldsymbol{K} q_{j}(t)=\boldsymbol{F}_{j}(t),
$$

where $\mathbf{M}$ - mass matrix, $\mathbf{B}$ - dumping matrix, $\mathbf{K}$ - stiffness matrix, $\mathbf{F}_{j}$ - generalized function of kinetic excitation. These matrixes are determined from:

$$
\begin{array}{cc}
\mathbf{M}=\mathbf{D} \mathbf{M}_{h}, & \mathbf{B}=\left(b_{i j}\right)=\left(d_{i i} \bar{b}_{i j}\right)=\mathbf{D}\left(d_{i i}\right) \mathbf{M}_{b}\left(\bar{b}_{i j}\right) \\
\mathbf{K}=\left(a_{i j}\right)=\left(d_{i i} \bar{a}_{i j}\right)=\mathbf{D}\left(d_{i i}\right) \mathbf{M}_{k}\left(\bar{a}_{i j}\right), & \mathbf{F}_{j}=\mathbf{D}\left(d_{\mathrm{ii}}\right) \cdot \mathbf{Q}_{j}
\end{array}
$$

And elements of diagonal matrix are

$$
d_{i i}=\frac{1}{\alpha_{i i}} \Rightarrow d_{11}=\frac{1}{m}, \quad d_{22}=\frac{1}{J_{x}}, \quad d_{33}=\frac{1}{J_{y}},
$$

where $m$ - mass.

Detailed and complete solution procedure is presented in the [1], including determination of particular coefficients. 
The system of simultaneous linear non-homogenous differential equations is determined by element definition of matrix $\mathbf{M}, \mathbf{B}, \mathbf{K}$ and components of vector $\mathbf{F}$. These equations are motion equations of the spatial vehicle model with three degrees of freedom, with total asymmetry: distribution of system mass $\left(e_{x} \neq 0, e_{y} \neq 0, D_{x y}=D_{y x} \neq 0\right)$, geometry and stiffness of the elastic support, geometry and intensity of viscous dumping and with asymmetry of kinetic excitation defined by roughness of the road $h(x) \rightarrow h(t)$.

It has to be pointed out, that the investigation of vehicle oscillation assuming the planar model with longitudinal axis of symmetry and especially under condition of quarter model with longitudinal and transversal axis of symmetry, essentially changes the definition of the matrix elements of the motion equations (7) and also the wanted solution. It can be proved by comparison of matrix elements of particular vehicle models.

For planar vehicle model with longitudinal axis of symmetry, i.g. for system with two degrees of freedom $w(t)$ and $\varphi_{y}(t)$ is valid

a) Mass distribution $D_{x y}=D_{y x}=0 \rightarrow S_{23}=\mathbf{S}_{32}=0, e_{y}=0$. Mass matrix $\mathbf{M}$ is diagonal, unitary,

b) Supports is given by dimensions $l_{y 1}=l_{y 4}, l_{y 2}=l_{y 3}, l_{x 1}=l_{x 4}, l_{x 2}=l_{x 3}$ (Fig. 1),

c) Dumping intensity $b_{1}=b_{4}, b_{2}=b_{3}$,

d) Stiffness of elastic support $k_{1}=k_{4}, k_{2}=k_{3}$.

Then the dumping matrix $\mathbf{B}$ elements are:

$$
\begin{array}{lll}
b_{11}=2 m^{-1}\left(b_{1}+b_{2}\right), & b_{12}=0, & \left.b_{13}=2 m^{-1}\left(b_{1} l_{x 1}-b_{2} l_{x 2}\right)-e_{x} b_{11}\right), \\
b_{21}=0, & b_{22}=J_{x}^{-1} 2 \sum_{j=1}^{3} b_{j} l_{y j}^{2}, & b_{23}=0, \\
b_{31}=m J_{x}^{-1} b_{13}, & b_{32}=0, & b_{33}=J_{y}^{-1}\left[2 \sum_{j=1}^{2} b_{j} l_{x j}^{2}+e_{x}^{2} b_{11} m\right] .
\end{array}
$$

And elements of stiffness matrix $\mathbf{K}$ :

$$
\begin{array}{lll}
a_{11}=2 m^{-1}\left(k_{1}+k_{2}\right), & a_{12}=0, & a_{13}=2 m^{-1}\left(k_{1} l_{x 1}-k_{2} l_{x 2}\right)-e_{x} \\
a_{21}=0, & a_{22}=2 J_{x}^{-1} \sum_{j=1}^{2} k_{j} l_{y j}^{2}, & a_{23}=0, \\
a_{31}=m J_{x}^{-1} a_{13}, & a_{32}=0, & a_{33}=J_{y}^{-1}\left[2 \sum_{j=1}^{2} k_{j} l_{x j}^{2}+e_{x}^{2} a_{11} m\right] .
\end{array}
$$

Components of vector of generalized function of the kinematic excitation for $h_{1}=h_{4}$, $h_{2}=h_{3}$ are as follows:

$$
\begin{gathered}
F_{1}(t)=2 m^{-1}\left[\sum_{j=1}^{2} k_{j} h_{j}(t)+\sum_{j=1}^{2} b_{j} \dot{h_{j}}(t)\right], \\
F_{2}(t)=0, \\
F_{3}(t)=J_{y}^{-1}\left[\sum_{m=1.4} \Phi_{m}(t) l_{x m}-\sum_{m=2.3} \Phi_{m}(t) l_{x m}+e_{x} \sum_{m=1}^{4} \Phi_{m}(t)\right],
\end{gathered}
$$


where $\Phi_{m}$ - vertical component of force in the location of $m$-th wheel between wheel and road, $k_{m}$ - spring stiffness of $m$-th wheel, $h_{m}$ - roughness height $m$-th wheel, $b_{m}$ - dumping of $m$-th wheel.

$$
\Phi_{m}(t)=k_{m} h_{m}(t)+b_{m} \dot{h}_{m}(t) .
$$

In this case, the first and the third equations from system (7) are simultaneous, the second equation is independent, homogenous equation of free oscillation.

For quarter model of the vehicle with longitudinal and transverse axis of symmetry, i.e. for system with one degree of freedom $w(t)$ is valid

a) Mass distribution $D_{x y}=D_{y x}=0 \rightarrow S_{23}=S_{32}=0, e_{x}=0, e_{y}=0$. Mass matrix $\mathbf{M}$ is unitary, gravity center is identical with geometrical center $\mathrm{T} \equiv \mathrm{C}$, the main central axes of inertia are identical with axes of geometrical symmetry,

b) Support geometry $l_{x j}=l_{x}, l_{y j}=l_{y}$ for $j=1,2,3,4$,

c) Dumping intensity $b_{j}=b$, for $j=1,2,3,4$,

d) Stiffness of elastic support $k_{j}=k$.

Elements of diagonal matrix of dumping $\mathbf{B}$ :

$$
\begin{array}{lll}
b_{11}=4 m^{-1} b, & b_{12}=0, & b_{13}=0, \\
b_{21}=0, & b_{22}=4 J_{x}^{-1} k l_{y}^{2}, & b_{23}=0, \\
b_{31}=0, & b_{32}=0, & b_{33}=4 J_{y}^{-1} k l_{x}^{2},
\end{array}
$$

and elements of diagonal stiffness matrix $\mathbf{K}$ :

$$
\begin{array}{lll}
a_{11}=4 m^{-1} k, & a_{12}=0, & a_{13}=0, \\
a_{21}=0, & a_{22}=4 J_{x}^{-1} k l_{y}^{2}, & a_{23}=0, \\
a_{31}=0, & a_{32}=0, & a_{33}=4 J_{y}^{-1} k l_{x}^{2} .
\end{array}
$$

Components of the vector of the generalized function of the kinematic excitation for $k_{j}=h$

$$
F_{1}(t)=4 m^{-1}[k h(t)+b \dot{h}(t)], \quad F_{2}(t)=0, \quad F_{3}(t)=0 .
$$

The difference of the individual vehicle models excel by rearrangement of the motion equations into the component form. The application of individual models is demonstrated by comparison which also indicates difference of solution difficulty [1]. The motion equations for followed models are compared in [1]:

- spatial generally symmetric model,

- spatial asymmetric model, but with parallel axes of inertia and geometric symmetry $\left(D_{x y}=D_{y x}=0\right)$,

- planar model with one axis of symmetry $\left(D_{x y}=D_{y x}=0, e_{y}=0\right)$.

Detailed solution of motion equations for all above mentioned cases is presented in the [1], including determination of particular coefficients.

\section{Results and discussion}

The procedure derived for simple 3D model was applied in many cases with analytical, numerical, simulation and experimental solution. The justification of possible application of the spatial solution was validated by analytical and numerical investigation of vertical 
vibration of the rigid plate supported on four springs with various degree of asymmetry (weights) and various kinematic excitation.

Symmetrical and asymmetrical distribution of the weights (geometrical asymmetry displacement of the gravity center $\mathrm{T}$ ) was solved for 6 basic cases ( 1 time symmetrical, 5 times asymmetrical) and 6 cases of asymmetrical excitation, assuming with and without dumping.

Kinematic excitation is realized by unit jump (Heaviside's function) $h=1$. This excitation is identical for all variants of model arrangement.

a) symmetrical $h_{1}=h_{2}=h_{3}=h_{4}=1$,

b) assymetrical

case

$$
\begin{array}{lll}
\text { 1. } & h_{1}=h_{4}=1 & h_{3}=h_{2}=0 \\
\text { 2. } & h_{1}=h_{2}=0 & h_{3}=h_{4}=1 \\
\text { 3. } & h_{1}=1 & h_{2}=h_{3}=h_{4}=0 \\
\text { 4. } & h_{1}=h_{2}=h_{4}=1 & h_{3}=0 \\
\text { 5. } & h_{1}=h_{3}=1 & h_{2}=h_{4}=0 .
\end{array}
$$

The vertical displacements of particular points of supports $\left(w_{1}, w_{2}, w_{3}, w_{4}\right)$, gravity center displacement $z_{T}$ and angles of axes rotations $\varphi_{x}, \varphi_{y}$ were determined. Due to range of the contribution, only selected results are presented. The symmetrical case with asymmetrical excitation (jump down of the wheel 1) with dumping is presented in the Fig 3.

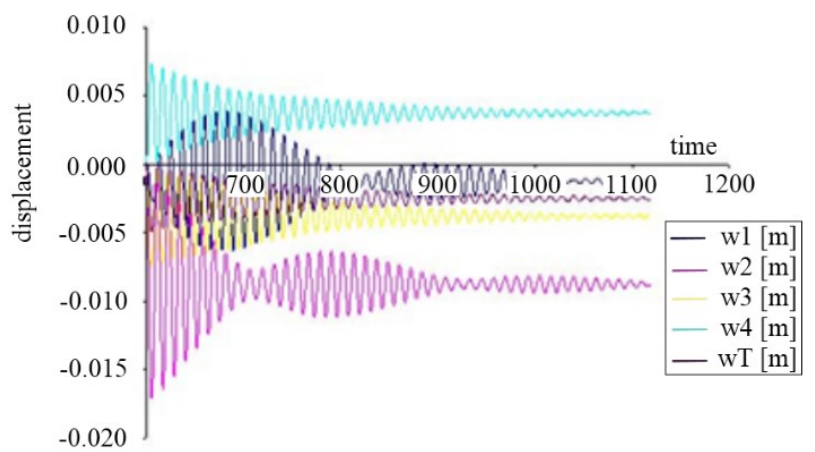

Fig. 3. Symmetrical model, asymmetrical excitation $3\left(h_{1}=1, h_{2}=h_{3}=h_{4}=0\right)$, movement of the gravity center $\mathrm{w}(\mathrm{t})$ and wheel (points) $\mathrm{w}_{1}(\mathrm{t}), \mathrm{w}_{2}(\mathrm{t}), \mathrm{w}_{3}(\mathrm{t})$, and $\mathrm{w}_{4}(\mathrm{t})$ with dumping

The same model without dumping is presented in the Fig 4.

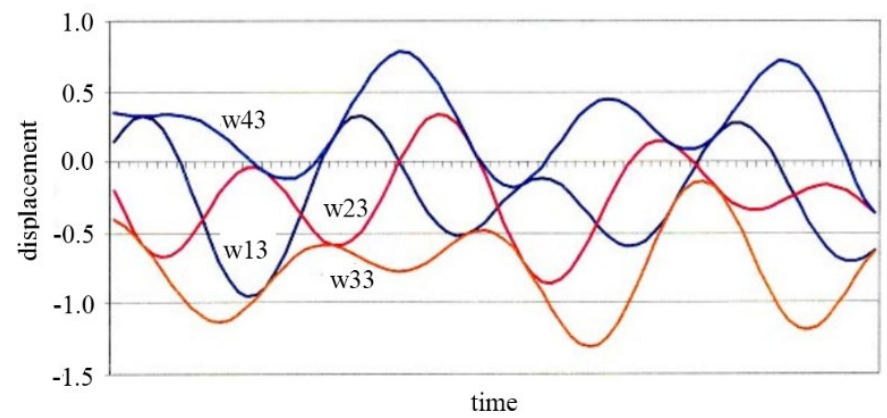


Fig. 4. Symmetrical model, asymmetrical excitation $3\left(h_{1}=1, h_{2}=h_{3}=h_{4}=0\right)-$ movement of wheels (points) 1-4 (w13, w23, w33, w43) without dumping

By the same methodology, the deviations (velocity, acceleration and axes rotation) were determined for all asymmetric models and symmetric model with combination of dumping presence or absence.

The asymmetric model, loaded by weight on the left half of the geometry with symmetrical excitation $\left(h_{1}=h_{2}=h_{3}=h_{4}=1\right)$ without dumping is presented in the Fig 5 .

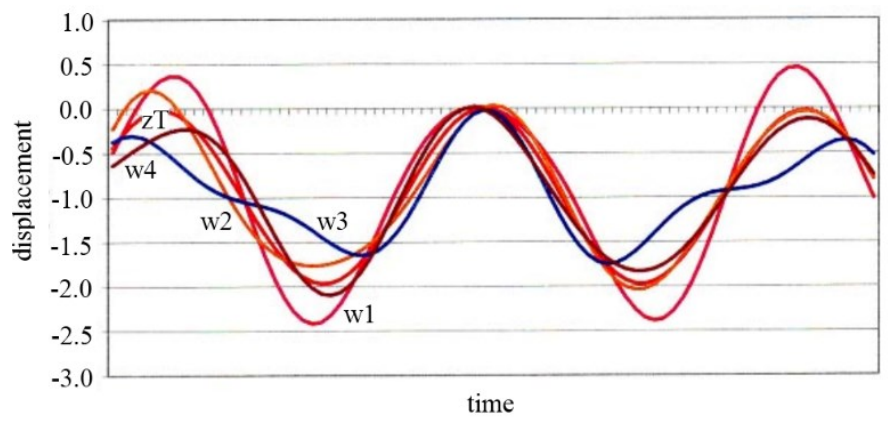

Fig. 5. Asymmetric model, symmetric excitation according to case 1, movement of the gravity center and points 1 to 4 without dumping

Similarly loaded plate with same asymmetric excitation as in the previous case but with dumping, is presented in the Fig 6.

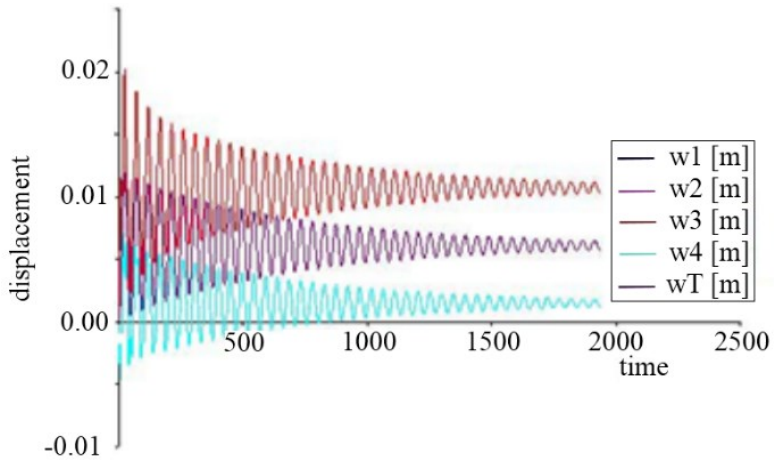

Fig. 6. Asymmetric model, symmetrical excitation of case 1, movement of gravity center and point 1 to 4 with dumping

It is very important to consider complete models (3D models) at investigation of vertical oscillation of bodies system with or without dumping, which arises from above mentioned text, because the asymmetry of mass distribution (and consequently eccentricity of gravity center from geometric center) as well as asymmetry of excitation or supports (differences between stiffness and dumping) are still present.

Beside simple 3D model, also the issues of vertical oscillation of body systems were investigated. One of these issues was e.g. vehicle passing over system of the road obstacles, where the system has 3 or 6 degrees of freedom. These issues are involve into the field of public transportation especially railway vehicles.

For investigation of the vertical displacement of the rail double-axle vehicle, it is possible to select rigid rectangular plate as the calculation model under certain assumptions. The rigid plate is loaded by asymmetric weights with, where the asymmetry position is regarded to geometrical axes of symmetry of median plane. The plate is spatially supported on four 
springs. Investigation of vertical displacements of plate for various cases of asymmetry under kinematic excitation of system with three degrees of freedom were presented in several contributions, see [6].

The vertical displacement of two-axle vehicle was solved analytically and further by FEM, respectively case of rigid body with effect of energy dissipation under vibration movements. The elastic support of the plate is combined with parallel viscous dumper. Scheme of the model is in the Fig 7.
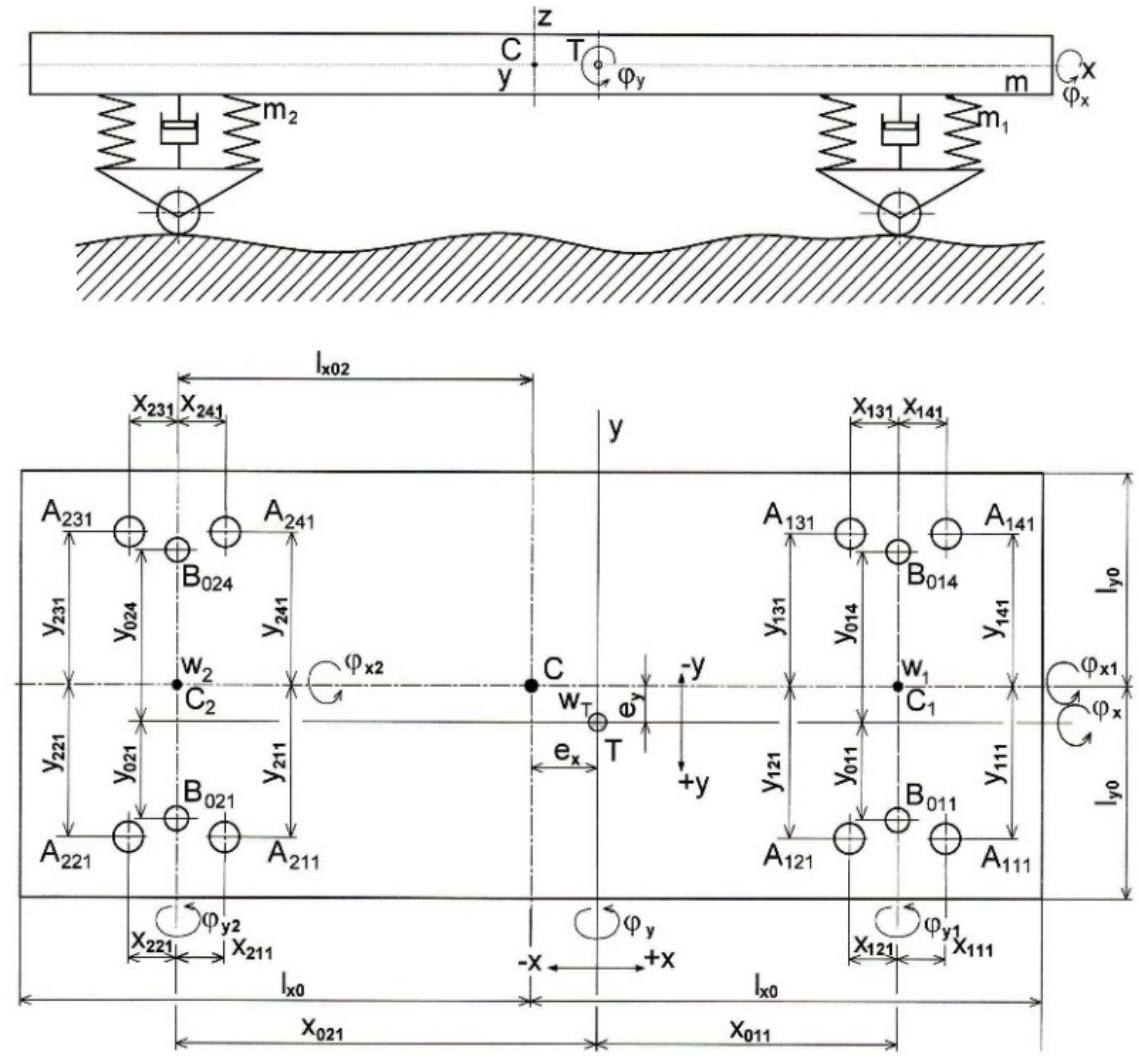

Fig. 7. Scheme of the railway two-axle vehicle model Fig. 7)

The motion equations can be expressed in the form for spatial support of the plate (see

$\left|\begin{array}{ccc}m & 0 & 0 \\ 0 & J_{x} & -D_{x y} \\ 0 & -D_{x y} & J_{y}\end{array}\right| \cdot\left|\begin{array}{l}\ddot{w} \\ \ddot{\phi}_{x} \\ \ddot{\phi}_{y}\end{array}\right|+\left|\begin{array}{ccc}\mu_{11} & \mu_{12} & \mu_{13} \\ \mu_{21} & \mu_{22} & \mu_{23} \\ \mu_{31} & \mu_{32} & \mu_{33}\end{array}\right| \cdot\left|\begin{array}{c}\dot{w} \\ \dot{\phi}_{x} \\ \dot{\phi}_{y}\end{array}\right|+\left|\begin{array}{ccc}\kappa_{11} & \kappa_{12} & \kappa_{13} \\ \kappa_{21} & \kappa_{22} & \kappa_{23} \\ \kappa_{31} & \kappa_{32} & \kappa_{33}\end{array}\right| \cdot\left|\begin{array}{c}w \\ \phi_{x} \\ \phi_{y}\end{array}\right|=\left|\begin{array}{l}F_{z}(t) \\ F_{x}(t) \\ F_{y}(t)\end{array}\right|,(9)$

where: $J_{x}, J_{y}$ are inertia moment and $D_{x y}$ is deviation moment to central axes of plate, $F_{z}(t)$, $F_{x}(t), F_{y}(t)$ are function of the external excitation including kinematic load. The system of equations (9) can be arranged to form:

$$
\left|\begin{array}{ccc}
1 & 0 & 0 \\
0 & 1 & s_{23} \\
0 & s_{32} & 1
\end{array}\right| \cdot\left|\begin{array}{l}
\ddot{y}_{1} \\
\ddot{y}_{2} \\
\ddot{y}_{3}
\end{array}\right|+\left|\begin{array}{ccc}
b_{11} & b_{12} & b_{13} \\
b_{21} & b_{22} & b_{23} \\
b_{31} & b_{32} & b_{33}
\end{array}\right| \cdot\left|\begin{array}{l}
\dot{y}_{1} \\
\dot{y}_{2} \\
\dot{y}_{3}
\end{array}\right|+\left|\begin{array}{lll}
k_{11} & k_{12} & k_{13} \\
k_{21} & k_{22} & k_{23} \\
k_{31} & k_{32} & k_{33}
\end{array}\right| \cdot\left|\begin{array}{l}
y_{1} \\
y_{2} \\
y_{3}
\end{array}\right|=\left|\begin{array}{l}
F_{1}(t) \\
F_{2}(t) \\
F_{3}(t)
\end{array}\right|
$$


where elements of the mass matrix $s_{23}=-D_{x y} / J_{x}, s_{32}=-D_{x y} / J_{y}$ determine effect of the asymmetry of mass displacement, respectively rotation of the main axes of inertia to central axes of inertia.

By gradual solution of the mentioned system of equations with application of Lagrange's transformation and by other arrangement the resulting equations for vertical displacement $y_{j}(t)$ can be obtained:

$$
\begin{aligned}
y_{j}(t)= & \sum_{i=1}^{3}(-1)^{i+j} \sum_{s=1}^{3}\left[K_{i s} \int_{o}^{t} F_{i}(\tau) \cdot e^{-\chi_{s}(t-\tau)} \cdot \cos \omega_{s}(t-\tau) \cdot d \tau+\right. \\
& \left.+\frac{L_{i s}-\chi_{s} K_{i s}}{\omega_{s}} \cdot \int_{0}^{t} F_{i}(\tau) \cdot e^{-\chi_{s}(t-\tau)} \cdot \sin \omega_{s}(t-\tau) \cdot d \tau\right] .
\end{aligned}
$$

Which is solution of (10), or by arrangement:

$$
\begin{array}{llrl}
y_{1}(t) & \Rightarrow w(t), & y_{2}(t) \Rightarrow \phi_{x}(t), & y_{3}(t) \Rightarrow \phi_{y}(t), \\
F_{1}(t) \Rightarrow F_{z}(t), & F_{2}(t) \Rightarrow F_{x}(t), & F_{3}(t) \Rightarrow F_{y}(t) .
\end{array}
$$

For case without dumping the equation compresses to the form

$$
y_{j}(t)=\sum_{i=1}^{3}(-1)^{j-i} \sum_{s=1}^{3} \frac{L_{i s}}{\omega_{s}} \int_{0}^{t} F_{i}(t) \cdot \sin \omega_{0 s}(t-\tau) \cdot d \tau .
$$

Detailed procedure of calculation is mentioned in [1].

\section{Conclusion}

The articles indicate the difficulty of the issue dealing with vertical oscillation of the system compounded from elastically bonded bodies with higher degrees of freedom. This issue is closely associated with contemporary problems of driving properties of vehicles, not only on-road but also railway. During driving of the vehicle, the unpredictable situations occur, when e.g. wrong distribution of the load can caused loss of driving stability due to random kinematic excitation (roughness on the road). The article brings the methodology how to investigate these situations and based on the result, the appropriate actions can be realized to eliminate material of life losses.

The work was supported by grant UJEP -SGS-2018-48-003-2.

\section{References}

1. J. Soukup, J. Volek et al., Vibration of mechanical systems - vehicles. Analysis of asymmetry effect. UJEP Ústí n. L., (2008)

2. M. Mitschke, Dynamik der Kraftfahrzeuge. Springer-Verlag, Berlin, Heidelberg, (1972)

3. K. Juliš, R. Brepta, et al., Mechanics II. Dynamcs. Technický průvodce 66, SNTL (1987)

4. R. Brepta, L. Půst, F. Turek, Mechanical oscillation. Technický průvodce Sobotáles 71 (1994) 
5. L. Rus, Dynamics of railroad vehicles. Ph.D. thesis, VÚML ČKD Praha, (1988)

6. J. Volek, J. Soukup, J. Kout, Investigation of influence of asymmetry in case of the vibration of resiliently supported plate, Engineering Mechanics, National conference 2003.

7. M. Blatnický, J. Dižo, M. Timoščuk, Design of a Three-Finger Robot Manipulator. 16, No 3, 485-489 (2016), ISSN 1213-2489

8. M. Loulová, A. Suchánek, J. Harušinec, Evaluation of the Parameters Affecting Passenger Riding Comfort of a Rail Vehicle. 17, No 2, 224-231 (2017), ISSN 12132489 